\title{
Acute pancreatitis: current perspectives on diagnosis and management
}

This article was published in the following Dove Press journal: Journal of Inflammation Research

\author{
Adarsh P Shah \\ Moustafa M Mourad \\ Simon R Bramhall \\ Department of Surgery, Hereford \\ County Hospital, Hereford, UK
}

Correspondence: Simon R Bramhall Department of Surgery, Hereford County Hospital, Stonebow Road, Hereford, UK, HRI 2ED

Email simon.bramhall@wvt.nhs.uk

\begin{abstract}
The last two decades have seen the emergence of significant evidence that has altered certain aspects of the management of acute pancreatitis. While most cases of acute pancreatitis are mild, the challenge remains in managing the severe cases and the complications associated with acute pancreatitis. Gallstones are still the most common cause with epidemiological trends indicating a rising incidence. The surgical management of acute gallstone pancreatitis has evolved. In this article, we revisit and review the methods in diagnosing acute pancreatitis. We present the evidence for the supportive management of the condition, and then discuss the management of acute gallstone pancreatitis. Based on the evidence, our local institutional pathways, and clinical experience, we have produced an outline to guide clinicians in the management of acute gallstone pancreatitis.
\end{abstract}

Keywords: acute pancreatitis, severity scoring, diagnostic imaging, management of gallstone pancreatitis

\section{Introduction}

A patient complaining of sudden onset of epigastric pain radiating to the back, associated with nausea and vomiting, requires rapid exclusion of a wide range of lifethreatening conditions involving the cardiovascular (myocardial infarction, ruptured, and/or dissecting aortic aneurysm) and gastrointestinal (peptic ulcer disease with perforation or bleeding, acute pancreatitis) systems. The clinician's history and examination findings are augmented by relevant investigations in narrowing the differential diagnoses to eventually guide the management and treatment of a certain condition and its associated complications.

The incidence of acute pancreatitis in the UK is $\sim 56$ cases per 100,000 persons per year, ${ }^{1}$ while in the US over 220,000 hospital admissions annually are attributed to acute pancreatitis. ${ }^{2}$ An epidemiologic study that utilized UK and European data demonstrated an increasing incidence in all-cause acute pancreatitis. ${ }^{3}$ The incidence of acute pancreatitis was also noted to increase with age. ${ }^{3,4}$ The male population had an incidence that was $10 \%-30 \%$ higher than females. ${ }^{4}$ Despite a reduction in the case fatality being observed over time, the population mortality has remained largely unchanged. ${ }^{3}$ Of all hospital admissions with acute pancreatitis, $\sim 20 \%-30 \%$ of patients have a severe course, ${ }^{1}$ while severe life-threatening complications will develop in $\sim 25 \%$ of these patients. ${ }^{4}$ The mortality in severe acute pancreatitis can be as high as $30 \%,{ }^{2}$ but the overall mortality in acute pancreatitis is estimated to be $5 \%{ }^{1}$ 
Gallstones remain the most common cause for acute pancreatitis. Gallstone-related acute pancreatitis accounts for approximately half of all UK cases, while up to $25 \%$ of acute pancreatitis cases can be attributed to alcohol. ${ }^{1}$ Epidemiologic data have shown a linear increase in the incidence of gallstone pancreatitis across the UK and European countries studied. However, the UK has a much lower incidence of alcohol-induced pancreatitis compared with European studies. ${ }^{3}$ Alcohol-induced acute pancreatitis is more common in middle-aged men. Idiopathic acute pancreatitis accounts for $20 \%-34 \%$ of cases and its incidence is similar in both men and women. ${ }^{3}$ The incidence of idiopathic acute pancreatitis depends on the extent to which a clinician investigates a patient's episode of acute pancreatitis for its causative etiology. Recent advances in laboratory pathology tests and radiologic imaging techniques have contributed to a reduction in the number of acute pancreatitis cases being labeled as idiopathic.

The incidence of gallstone-related acute pancreatitis in both men and women increases with age, with women over the age of 60 years at higher risk., ${ }^{2,3}$ Patients with gallstones smaller than $5 \mathrm{~mm}$, microlithiasis, or biliary sludge are thought to be at higher risk of gallstone pancreatitis. Microlithiasis causes a functional obstruction at the sphincter of Oddi, which subsequently results in bile and/or biliarypancreatic secretion reflux that injures the pancreatic duct. ${ }^{5}$ The common channel theory in the pathogenesis of acute gallstone pancreatitis has been refuted by some. ${ }^{6}$ Instead, it has been postulated that acute gallstone pancreatitis is the result of pancreatic acinar hyperstimulation secondary to ductal obstruction that triggers trypsin release, which induces a cascade of enzyme-led pancreatic and peripancreatic inflammation. ${ }^{6}$ Others speculate that duodenal content reflux is more causative of pancreatic ductal injury than bile reflux. ${ }^{7}$ There are multiple theories implicated in the pathogenesis of acute pancreatitis, and all remain controversial.

Inappropriate release and activation of pancreatic enzymes induce acute pancreatitis. The key enzyme in the activation of pancreatic zymogens has been thought to be trypsin. The inappropriate activation of trypsinogen to trypsin and the lack of prompt pancreatic clearance of active trypsin result in pancreatic inflammation and subsequent triggering of the inflammatory cascade. ${ }^{2}$ Cytokines including interleukin (IL)-1, IL-6, IL-8, tumor necrosis factor $\alpha$, and platelet-activating factor are released. ${ }^{7}$ These in turn induce the hepatic synthesis of acute phase reaction proteins such as $\mathrm{C}$-reactive protein (CRP). Leukocyte migration and activation may represent the major determining factor for both local and systemic complications. ${ }^{4}$

\section{Diagnosis of acute pancreatitis}

In their 2005 guidelines, the UK Working Party on Acute Pancreatitis suggested that the etiology should be determined in at least $80 \%$ of cases of acute pancreatitis. Furthermore, the classification of cases of idiopathic acute pancreatitis should be no more than $20 \% .{ }^{8}$ Therefore, patients are subjected to extensive investigations to determine the underlying etiology.

The pretest probability of acute pancreatitis is determined by the clinician's index of suspicion, which is largely based on the patient's history and clinician's examination findings. ${ }^{4}$ The classical teaching is that a serum amylase level that is three or four times greater than the upper limit of normal is diagnostic of acute pancreatitis. While the measurement of serum pancreatic enzymes such as amylase is the "gold standard" for the diagnosis of acute pancreatitis, the measured value for the serum pancreatic enzymes should be interpreted by considering the duration of patient's symptoms.

In acute pancreatitis, the pancreatic enzymes amylase, lipase, elastase, and trypsin are simultaneously released into the bloodstream. As the clearance of each of these enzymes varies, the timing of the blood sampling from the onset of acute pancreatitis affects the test's sensitivity. ${ }^{4}$ Lipase has a higher diagnostic accuracy compared to amylase as the serum lipase levels are elevated for a longer period. ${ }^{9}$ Caution should be exercised when interpreting amylase results in patients with hypertriglyceridemia as they can have a falsely low amylase result.

During an attack of acute pancreatitis, the elevation of alanine aminotransferase to $>150 \mathrm{IU} / \mathrm{L}$ is a predictive factor for biliary cause of acute pancreatitis. ${ }^{10} \mathrm{~A}$ previous metaanalysis has indicated that this threefold elevation in alanine aminotransferase has a positive predictive value of $95 \%$ in diagnosing acute gallstone pancreatitis. ${ }^{11}$

The biochemical measurement of trypsinogen activation peptide (TAP) and trypsinogen-2 is more useful as a diagnostic marker for acute pancreatitis due to their accuracy, but their evaluation is limited by availability. ${ }^{9}$ Early elevated levels of urinary TAP have been shown to be associated with severe acute pancreatitis. ${ }^{4}$ Other markers such as IL-6 and IL- $8,{ }^{9}$ as well as phospholipase A2 have been summarized well elsewhere, ${ }^{12}$ and are not routinely measured in clinical practice in the UK.

\section{Management of acute pancreatitis Classification of severity}

Mastery of the management of acute pancreatitis is an art that can challenge experienced clinicians at the best of 
times. One facet to the art of managing acute pancreatitis is classification of the disease severity so that one can recognize, anticipate, and treat accordingly complications of the disease. The revised 2012 Atlanta criteria for classification of the severity of acute pancreatitis are widely accepted. ${ }^{13}$ This revised classification defines transient organ failure as organ failure which resolves completely within 48 hours, whereas failure of resolution of organ failure is defined as persistent. The presence of persistent organ failure, usually with one or more local complications, indicates severe acute pancreatitis. On the other hand, the absence of organ failure without any local or systemic complications indicates mild acute pancreatitis. "Moderately severe acute pancreatitis", indicated by transient organ failure and/or local or systemic complications in the absence of persistent organ failure, is the new grade of severity between mild and severe that was introduced in the revised classification. ${ }^{13}$ Multiple scoring systems for the prediction of the disease severity and prognostic implications exist. ${ }^{12,14}$ The prognostic features aid the clinician in predicting complications of acute pancreatitis. ${ }^{8}$

The Acute Physiology and Chronic Health Evaluation (APACHE) II scoring system has demonstrated the highest accuracy for predicting severe acute pancreatitis when compared with other scoring systems. ${ }^{15}$ Other markers of severe acute pancreatitis based on evidence from the literature have been outlined in Box 1. The APACHE II score can be repeated

Box I The clinical, biochemical, and radiologic markers that assist in the classification of severity of an acute pancreatitis episode.

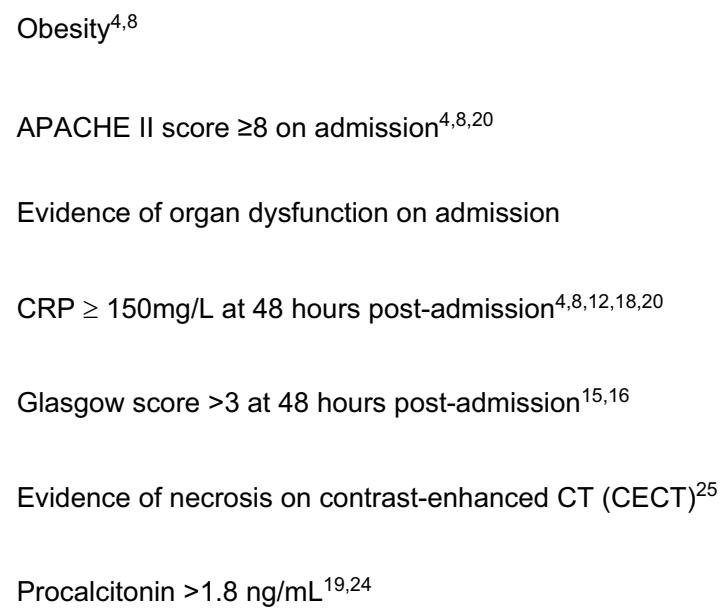

Notes: These aid the clinician with identifying patients who should have early intensive care input or treatment. Patients with any combination of the above should be classed as severe acute pancreatitis and thus monitored for complications within an escalated level of care.

Abbreviations: APACHE, Acute Physiology and Chronic Health Evaluation; CRP, C-reactive protein. daily and its trends correlate well with clinical progress or deterioration. However, there is no significant difference in the prognostic accuracy between the APACHE II and multiple factor scoring systems such as Ranson, computed tomography severity index (CTSI), ${ }^{15,16}$ and the bedside index for severity in acute pancreatitis. ${ }^{17}$

The CRP is a reliable, easily accessible, single marker of assessing severity. It has demonstrated good prognostic accuracy for severe acute pancreatitis, pancreatic necrosis, and in-hospital mortality when measured at 48 hours following hospital admission. ${ }^{18,19}$ Another cheap and easily obtainable parameter indicative of the severity of acute pancreatitis is the hematocrit. An admission hematocrit $\geq 44 \%$ or failure of the hematocrit to decrease at 24 hours following admission is indicative of severe acute pancreatitis in the early stage of the disease. ${ }^{20}$ Additionally, some studies have demonstrated that hemoconcentration has been associated with the risk of developing necrotizing pancreatitis and organ failure, ${ }^{20,21}$ while others refute this observation. ${ }^{22,23}$ The absence of hemoconcentration on admission has a high negative predictive value for the development of necrosis., ${ }^{22,23}$ Other markers such as procalcitonin ${ }^{19,24}$ and IL-8, not used routinely in the UK, have been shown to have high predictive accuracy in classifying the severity of necrotizing pancreatitis in the first days of the disease.

The inflammatory response varies between each individual patient. The release of intrapancreatic enzymes triggers the release of proinflammatory mediators and macrophage activation within acinar cells resulting in local complications of acute pancreatitis, which include pancreatic necrosis with or without infection, pancreatic pseudocyst formation, pancreatic duct disruption, and peripancreatic vascular complications. It is unclear why in some patients the local pancreatic inflammation triggers a systemic release of proinflammatory mediators. However, this systemic inflammatory response manifests as organ failure, and its recognition and treatment are important in altering the clinical course of acute pancreatitis.

\section{Imaging}

Imaging plays an important role in the diagnosis and management of acute pancreatitis. As $50 \%$ of acute pancreatitis cases are gallstone-related, transabdominal ultrasound is the most common initial radiologic investigation of choice. Ultrasonography has the highest sensitivity for detection of gallbladder stones, but a poor sensitivity for choledocholithiasis (Table 1). The retroperitoneally sited pancreas is usually difficult to visualize in acute pancreatitis during 
Table I Comparison of the different imaging modalities available when diagnosing choledocholithiasis

\begin{tabular}{llllll}
\hline Imaging modality & Sensitivity (\%) & $\begin{array}{l}\text { Specificity } \\
(\%)\end{array}$ & $\begin{array}{l}\text { Positive predictive } \\
\text { value (\%) }\end{array}$ & $\begin{array}{l}\text { Negative predictive } \\
\text { value (\%) }\end{array}$ & $\begin{array}{l}\text { Accuracy } \\
\text { (\%) }\end{array}$ \\
\hline Transabdominal ultrasound & $50-80^{30}$ & $90^{30}$ & $100^{10}$ & $80^{10}$ & $92-99^{34}$ \\
Endoscopic ultrasound & $84-100^{30,31}$ & $94-100^{32,33}$ & $98^{31}$ & $88^{31}$ & $94^{34}$ \\
CECT & $60-88^{30,34}$ & $97-100^{30,34}$ & & & $95.2^{30,35}$ \\
MRCP & $81-100^{30,34,35}$ & $72-98^{30,34,35}$ & $90.5^{30,35}$ & $84^{30,34,35}$ \\
ERCP & $89^{31}$ & $100^{31}$ & $100^{31}$ & $83^{31}$ & \\
\hline
\end{tabular}

Abbreviations: CECT, contrast-enhanced CT; ERCP, endoscopic retrograde cholangiopancreatography; MRCP, magnetic resonance cholangiopancreatography.

Box 2 The benefits of magnetic resonance imaging in acute pancreatitis. ${ }^{36}$

- Nonionizing radiation - useful in following up patients with repeated scans;

- Ability to detect choledocholithiasis;

- Ability to demonstrate the presence of a disconnected pancreatic duct useful in predicting complications and of some prognostic value;

- Ability to characterize pancreatic and parenchymal collections or abscesses detecting complications of acute pancreatitis, therefore guiding management;

- With angiography, useful in identifying hemorrhage within pancreatic or peripancreatic collections or pseudocysts, and identifying pseudoaneurysms to guide further management.

ultrasonography, which can be further compounded by overlying bowel gas, large patient body habitus, and abdominal pain. In the assessment of the presence or absence of gallstones, it is recommended that at least two good quality ultrasound examinations are obtained. Where the first exam is negative and cannot detect gallstones, the most sensitive test for diagnosis of gallstones that may have been initially missed remains a further ultrasound examination. ${ }^{4}$

In patients with suspected acute pancreatitis, dynamic contrast-enhanced CT (CECT) is the imaging modality of choice. CECT plays a role in establishing the diagnosis, staging the severity of the disease, and assists in the detection of complications. ${ }^{4,25}$ However, it must be borne in mind that the staging of severity and detection of complications depend on the timing of CT scanning. In the first 24-48 hours, the CT findings of necrosis may be equivocal as only $25 \%$ of patients with acute pancreatitis develop necrosis. Additionally, pancreatic necrosis may not develop within the first 48 hours. ${ }^{20}$ In severe acute pancreatitis, unless the patient is critically ill and in need of emergency intervention, the initial CT scan should ideally be obtained at least 72 hours following symptom onset. ${ }^{4}$
The use of CECT in the localization of site and/or extent of pancreatic necrosis enhances the accuracy in outcome prediction, as evident from the development of the CTSI. High CTSI scores correlate with worsening severity and prognosis, pancreatic infection, and need for intervention. ${ }^{26,27}$ For example, patients with necrosis of the pancreatic head have similar poor outcomes in comparison to patients whose entire pancreas was affected. ${ }^{28}$ A modified CTSI has been developed for evaluating the severity of acute pancreatitis, but no significant differences have been observed when compared to the original CTSI. However, both CTSI scoring systems have demonstrated superior accuracy in diagnosing clinically severe acute pancreatitis when compared to the APACHE II severity scoring system. ${ }^{29}$

Magnetic resonance imaging in the form of magnetic resonance cholangiopancreatography (MRCP) has become a popular imaging modality for evaluation of the bile ducts and pancreatic duct. Its benefits in acute pancreatitis are outlined in Box 2. MRCP is reliable in diagnosing choledocholithiasis, and is only superseded by endoscopic ultrasound (EUS) in its sensitivity for detecting choledocholithiasis (Box 2). The limitations of MRCP include contraindication in patients with 
pacemakers and other metal objects, long image acquisition times, and difficulty with scanning critically ill patients.

The advent of MRCP and EUS has dramatically reduced the need for ERCP as a diagnostic tool in choledocholithiasis. EUS is the most reliable pretherapeutic diagnostic modality for choledocholithiasis, ${ }^{34}$ and when utilized with MRCP, both imaging modalities provide a safer method for investigating choledocholithiasis compared to ERCP, which is itself associated with a risk of postprocedural pancreatitis. EUS is particularly useful in the assessment of microlithiasis, which has been attributed as a cause of recurrent acute pancreatitis in patients with no evidence of choledocholithiasis visible using other imaging modalities. EUS also confers the ability to evaluate ductal abnormalities.

\section{Fluid therapy in acute pancreatitis}

The initial management of acute pancreatitis is largely supportive, with fluid replacement and optimization of electrolyte balance, providing adequate caloric support, and preventing or identifying and treating local and systemic complications.

The local and systemic inflammatory response in acute pancreatitis results in fluid depletion in the form of vomiting, reduced oral fluid intake, third-space fluid loss, and increased insensible losses in sweat and respiration. Fluid replacement in acute pancreatitis can be undertaken using crystalloid, colloid, or a combination of both. Ringer's lactate is the preferred crystalloid fluid, ${ }^{37}$ but caution should be exercised in hypercalcemic patients. The literary evidence for recommendations for fluid resuscitation has been summarized previously. ${ }^{38}$ However, to date, there is no clear agreed consensus regarding the ideal fluid type and regimen for fluid resuscitation. ${ }^{39,40}$ The goal of fluid resuscitation is to achieve a urine output of $\geq 0.5 \mathrm{~mL} / \mathrm{kg} / \mathrm{h}$ and a target heart rate $<120 / \mathrm{min}$, and maintain hematocrit between $35 \%$ and $44 \%$. ${ }^{39}$ Supplemental oxygen should complement fluid resuscitation to maintain arterial oxygen saturations $>95 \%$.

\section{Nutrition in acute pancreatitis}

Acute pancreatitis results in the rapid metabolism of fat and protein due to the hypercatabolic state. Nutritional support aims to provide adequate caloric intake and modulate the oxidative stress response during the initial phase of acute pancreatitis, thereby counteracting the catabolic effects. ${ }^{41}$ Additionally, enteral nutrition maintains intestinal motility, which preserves the gut barrier function and subsequently reduces the risk of secondary infections - it has been hypothesized that the infective complications of acute pancreatitis arise because of bacterial translocation from the gut, a consequence of altered intestinal motility, bacterial overgrowth, and increased intestinal permeability. ${ }^{39}$ Enteral nutrition, compared to total parenteral nutrition, in acute pancreatitis is associated with better clinical outcomes. ${ }^{42}$

Immediate oral feeding with the introduction of a soft diet, ${ }^{43}$ low-fat solid diet, ${ }^{44}$ or a full solid diet $^{45}$ is safe in patients with mild acute pancreatitis whose pain is settling. Oral feeding in mild acute pancreatitis has been shown to result in shorter duration of hospitalization with no significant pain relapse noted after initiation of refeeding. ${ }^{43,45}$ However, caution should be exercised in patients whose pain relapses following early oral refeeding, as this has been shown to increase their hospital stay. ${ }^{46}$

In patients with severe acute pancreatitis, there is good evidence that enteral nutrition is preferred over total parenteral nutrition. Total parenteral nutrition is associated with a significant increase in local and systemic infective complications, multiorgan failure, and mortality. ${ }^{42,47,48}$ Enteral nutrition within 48 hours of admission modulates the inflammatory and sepsis response, ${ }^{41,49}$ which has demonstrated clinically significant reduction in all infections and mortality in one study.$^{50}$ However, two randomized clinical trials comparing early enteral feeding vs delayed enteral feeding in acute severe pancreatitis failed to demonstrate superiority of early enteral feeding in reducing complications, organ failure, and mortality in these patients. ${ }^{51,52}$ Enteral feeding can be administered via the nasogastric and nasojejunal routes. Nasogastric feeding is safe and has comparable outcomes to nasojejunal feeding in severe acute pancreatitis. ${ }^{53,54}$ The UK Working Party on Acute Pancreatitis recommends the use of the enteral route for nutritional support, if tolerated, in patients with severe acute pancreatitis. It also acknowledges that the evidence to support the use of enteral nutrition in all patients with severe acute pancreatitis is not conclusive. ${ }^{8}$

\section{Antibiotic therapy in acute pancreatitis}

Secondary infective complications of acute pancreatitis are associated with increased mortality. ${ }^{8}$ The widespread use of antimicrobial therapy across all areas of health care has resulted in the need for targeted antimicrobial therapy to achieve better outcomes while simultaneously minimizing the risk of developing antimicrobial resistance. Like the debate surrounding nutrition in acute pancreatitis, there have been controversies with the use of antimicrobials in acute pancreatitis.

The spectrum of microorganisms responsible for infected necrosis is changing. ${ }^{4}$ Although Gram-negative aerobic 
bacteria are commonly yielded in cultures of infected pancreatic necrosis, Gram-positive bacteria, anaerobes, and fungi have also been isolated. ${ }^{55}$ Penicillins, first-generation cephalosporins, aminoglycosides, and tetracyclines are ineffective in acute pancreatitis. Antibiotics that are active against Gramnegative bacteria such as imipenem, clindamycin, piperacillin, fluoroquinolones, and metronidazole have adequate tissue penetration and bactericidal properties in infected pancreatic necrosis. ${ }^{56}$ Compared with other intravenous antibiotics, carbapenems are associated with a significant reduction in mortality, while use of imipenem significantly reduced the incidence of infected pancreatic necrosis. ${ }^{55,57}$ Caution should be exercised when interpreting results of the meta-analyses as the patient numbers are relatively small. ${ }^{57,59}$

Studies conducted two decades ago focused on the use of antibiotic prophylaxis in preventing the infective complications in severe acute pancreatitis. Systematic reviews and meta-analysis of these studies demonstrated a reduction in mortality with antibiotic prophylaxis. ${ }^{58,59}$ Two decades later, there is now good evidence to suggest that there is no significant decrease in mortality when patients with acute pancreatitis are treated with prophylactic antibiotics. Furthermore, prophylactic antibiotics are not associated with a significant reduction in infective complications of pancreatic necrosis. ${ }^{57,60,61}$

\section{Surgical intervention in acute pancreatitis}

The surgical management for acute pancreatitis can be divided into the surgical management of acute gallstone pancreatitis and the surgical management of complications of acute pancreatitis. Figure 1 summarizes the management of mild acute pancreatitis, including cases whose etiology is related to gallstones.

In patients with mild acute gallstone pancreatitis who are fit for cholecystectomy, the guidelines recommend that the procedure should ideally be performed at the index admission, ${ }^{62}$ and should not be delayed by $>2$ weeks. ${ }^{8,63}$ Early laparoscopic cholecystectomy in this cohort of patients can shorten the total hospital stay. ${ }^{64}$ On the other hand, studies involving patients with mild acute gallstone pancreatitis who underwent interval (delayed) cholecystectomy observed a high risk of readmission with recurrent biliary events. ${ }^{8,65}$ For patients who are at high risk or unfit for cholecystectomy, or in centers where in-patient cholecystectomy during the index admission is not a feasible option, ERCP and endoscopic sphincterotomy (ES) alone may be sufficient. ES can

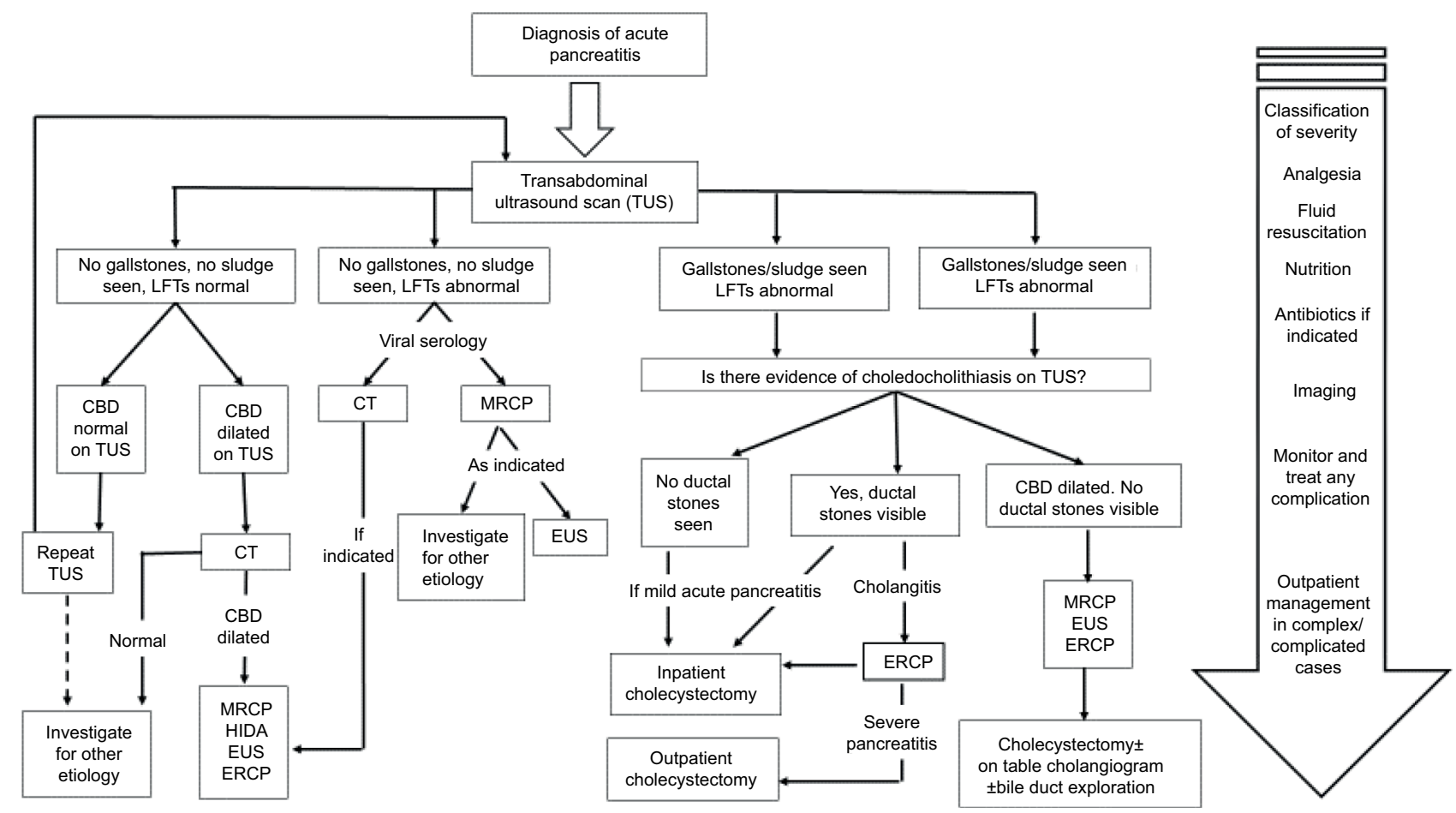

Figure I A flowchart encompassing the patient's journey from diagnosis of acute pancreatitis through to further investigation and definitive management.

Abbreviations: CBD, common bile duct; CT, computed tomography; ERCP, endoscopic retrograde cholangiopancreatography; EUS, endoscopic ultrasound; HIDA, fatty meal hepatobiliary iminodiacetic acid scan; LFT, liver function test; MRCP, magnetic resonance cholangiopancreatography; TUS, transabdominal ultrasound. 
reduce the short-term risk of a second attack of pancreatitis by at least $50 \%{ }^{4}$

All patients with acute gallstone pancreatitis should have imaging of the common bile duct to assess for choledocholithiasis (Box 2). Preoperative imaging utilizes noninvasive methods such as transabdominal ultrasound and/or MRCP, while intraoperative cholangiography provides real-time imaging of the common bile duct. Management of choledocholithiasis is reliant upon availability of local expertise and can be broadly classified into 1) the single-stage approach laparoscopic or open cholecystectomy with intraoperative cholangiography and common bile duct exploration, or 2) two-stage approach - preoperative ERCP with or without ES followed by laparoscopic or open cholecystectomy. There is no significant difference in the morbidity, mortality, retained stones, and failure rate between the two management approaches for choledocholithiasis. ${ }^{66,67}$

In centers where the availability of appropriate surgical expertise allows the single-stage definitive management of mild acute gallstone pancreatitis, promising results have been yielded. Low complication and conversion rates have been observed, although the selection of patients with uncomplicated mild acute gallstone pancreatitis may account for this. ${ }^{68,69}$ Postoperative MRCP and/or ERCP are options available to clinicians should there be any concern regarding retained stones or alternative pathologies. ${ }^{68}$ The combination of laparoscopic cholecystectomy and preoperative ES has also demonstrated a safe and reliable approach in dealing with choledocholithiasis during acute gallstone pancreatitis. ${ }^{70}$

In patients with severe acute gallstone pancreatitis with choledocholithiasis and/or cholangitis, the evidence suggests that performing ERCP within 72 hours of admission reduces the morbidity and mortality in this group of patients. ${ }^{71}$ Furthermore, ERCP reduces the length of hospital stay in patients with acute severe gallstone pancreatitis. To date, there is no evidence for or against early laparoscopic cholecystectomy for patients with severe acute gallstone pancreatitis.

Local complications of acute pancreatitis include pancreatic necrosis with or without infection, pancreatic pseudocyst formation, pancreatic duct disruption, and peripancreatic vascular complications. These local complications can be managed using a combination of endoscopic, radiologic, and surgical techniques, and have been reviewed previously. ${ }^{72}$ Open surgical debridement requires multiple laparotomies and is consequently associated with a high postoperative morbidity. However, surgical techniques have evolved to become minimally invasive, which may be associated with better outcomes. ${ }^{73}$
The "skunk procedure" utilizes imaging to advance catheters (drains) over their guidewires into the infected area within the lesser sac. Closed continuous lavage is then initiated once the catheters are in a satisfactory position. ${ }^{74}$ The drainage catheters facilitate a pathway into the lesser sac when performing a video-assisted minimally invasive retroperitoneal pancreatic necrosectomy. Endoscopic transgastric or transduodenal drainage of infected pancreatic necrosis or pancreatic pseudocysts is another technique associated with lower morbidity than open surgical debridement. The minimally invasive techniques for dealing with complications of acute pancreatitis require a multidisciplinary approach with specialist personnel, skills, and equipment. The procedures should ideally be undertaken in centers where there is readily available expertise to manage any complications.

\section{Conclusion}

Acute pancreatitis is frequently encountered on the emergency surgical take. Once the diagnosis is made, clinical efforts should simultaneously concentrate on investigating for the underlying etiology and managing the condition by anticipating its complications, which can be aided by using any of the severity scoring systems described. Management of acute pancreatitis is largely supportive. There is still no consensus on the ideal type and regimen of fluid for resuscitation, but goal-directed fluid therapy is associated with better outcomes. Early enteral nutrition modulates the inflammatory response and improves outcomes by decreasing infective complications of acute pancreatitis. Antibiotics should be used judiciously as prophylactic antibiotics have not shown any benefit in preventing infective complications of acute pancreatitis. Patients with mild acute gallstone pancreatitis should be recommended to undergo a laparoscopic cholecystectomy at the index admission, while those with severe gallstone pancreatitis and evidence of cholangitis and/or choledocholithiasis benefit from early ERCP. Patients with mild acute gallstone pancreatitis and concurrent choledocholithiasis benefit from single-stage laparoscopic cholecystectomy and bile duct exploration, subject to available local expertise. There is no difference in mortality and morbidity between the single-stage and double-stage management of choledocholithiasis. However, the single-stage approach reduces the length of hospital stay and need for recurrent admissions.

\section{Disclosure}

The authors report no conflicts of interest in this work. 


\section{References}

1. NICE. Pancreatitis: Diagnosis and Management Draft Scope for Consultation. National Institute for Clinical Excellence: London, UK; 2016.

2. Whitcomb DC. Acute pancreatitis. $N$ Engl J Med. 2006;354(20): 2142-2150.

3. Yadav D, Lowenfels AB. Trends in the epidemiology of the first attack of acute pancreatitis. Pancreas. 2006;33(4):323-330.

4. Toouli J, Brooke-Smith M, Bassi C, et al. Guidelines for the management of acute pancreatitis. J Gastroenterol Hepatol. 2002;17:515-539.

5. Venneman NG, van Brummelen SE, van Berge-Henegouwen P, van Erpecum KJ. Microlithiasis: an important cause of "idiopathic" acute pancreatitis? Ann Hepatol. 2003;2(1):30-35.

6. Wang GJ, Gao CF, Wei D, Wang C, Ding SQ. Acute pancreatitis: etiology and common pathogenesis. World J Gastroenterol. 2009;15(12):1427-1430.

7. Sakorafas GH, Tsiotou AG. Etiology and pathogenesis of acute pancreatitis: current concepts. J Clin Gastroenterol. 2000;30(4):343-356.

8. UK Working Party on Acute Pancreatitis. UK guidelines for the management of acute pancreatitis. Gut. 2005;54(Suppl 3):1-9.

9. Matull WR, Pereira SP, O'Donohue JW. Biochemical markers of acute pancreatitis. J Clin Pathol. 2006;59(4):340-344.

10. Ammori B, Boreham B, Lewis P, Roberts S. The biochemical detection of biliary etiology of acute pancreatitis on admission: a revisit in the modern era of biliary imaging. Pancreas. 2003;26(2):e32-e35.

11. Tenner S, Dubner H, Steinberg W. Predicting gallstone pancreatitis with laboratory parameters: a meta-analysis. Am J Gastroenterol. 1994;89(10):1863-1866.

12. Carroll J, Herrick B, Gipson T, Lee S. Acute pancreatitis: diagnosis, prognosis and treatment. Am Fam Physician. 2007;75(10):1513-1520.

13. Banks PA, Bollen TL, Dervenis C, et al. Classification of acute pancreatitis 2012: revision of the Atlanta classification and definitions by international consensus. Pancreas. 2013;62(1):102-111.

14. BMJ. Acute pancreatitis. BMJ Best Practice. 2017. Available from: http://bestpractice.bmj.com/best-practice/monograph/66/diagnosis/ criteria.html. Accessed October 2017.

15. Cho JH, Kim TN, Chung HH, Kim KH. Comparison of scoring systems in predicting the severity of acute pancreatitis. World J Gastroenterol. 2015;21(8):2387-2394.

16. Wilson C, Heath DI, Imrie CW. Prediction of outcome in acute pancreatitis: a comparative study of APACHE II, clinical assessment and multiple factor scoring systems. Br J Surg. 1990;77(11):1260-1264.

17. Papachristou GI, Muddana V, Yadav D, et al. Comparison of BISAP, Ranson's, APACHE II, and CTSI scores in predicting organ failure, complications, and mortality in acute pancreatitis. Am J Gastroenterol. 2010;105(2):435-441.

18. Cardoso F, Ricardo L, Oliveira A, et al. C-reactive protein prognostic accuracy in acute pancreatitis: timing of measurement and cut-off points. Eur J Gastroenterol Hepatol. 2013;25(7):784-789.

19. Staubli S, Oertli D, Nebiker C. Laboratory markers predicting severity of acute pancreatitis. Crit Rev Clin Lab Sci. 2015;52(6):273-283.

20. Berger HG, Rau BM. Severe acute pancreatitis: clinical course and management. World J Gastroenterol. 2007;13(38):5043-5051.

21. Muddana V, Whitcomb D, Khalid A, Slivka A, Papachristou G. Elevated serum creatinine as a marker of pancreatic necrosis in acute pancreatitis. Am J Gastroenterol. 2009;104(1):164-170.

22. Lankisch P, Mahlke R, Blum T, Bruns A, Maisonneuve P, Lowenfels A. Hemoconcentration: an early marker of severe and/or necrotizing pancreatitis? A critical appraisal. Am J Gastroenterol. 2001;96(7):2081-2085.

23. Gardner T, Olenec C, Chertoff J, Mackenzie T, Robertson D. Hemoconcentration and pancreatic necrosis: further defining the relationship. Pancreas. 2006;33(2):169-173.

24. Kylanpaa-Back ML, Takala A, Kemppainen E, Puolakkainen P, Haapiainen R, Repo H. Procalcitonin strip test in the early detection of severe acute pancreatitis. Br J Surg. 2001;88(2):222-227.

25. Balthazar E. CT diagnosis and staging of acute pancreatitis. Radiol Clin North Am. 1989;27(1):19-37.
26. Balthazar E, Robinson D, Megibow A, Ranson J. Acute pancreatitis: value of CT in establishing prognosis. Radiology. 1990;174(2):331-336.

27. Simchuk EJ, Traverso LW, Nukui Y, Kozarek RA. Computed tomography severity index is a predictor of outcomes for severe pancreatitis. Am J Surg. 2000;179(5):352-355.

28. Wyncoll DL. The management of severe acute necrotising pancreatitis: an evidence-based review of the literature. Intensive Care Med. 1999;25:146-156.

29. Bollen T, Singh V, Maurer R, et al. Comparative evaluation of the modified CT severity index and CT severity index in assessing severity of acute pancreatitis. Am J Roentgenol. 2011;11(2):386-392.

30. Surlin V, Saftoiu A, Dumitrescu D. Imaging tests for accurate diagnosis of acute biliary pancreatitis. World $J$ Gastroenterol. 2014;20(44):16544-16549.

31. Prat F, Amouyal G, Amouyal P, et al. Prospective controlled study of endoscopic ultrasonography and endoscopic retrograde cholangiography in patients with suspected common-bileduct lithiasis. Lancet. 1996;347(8994):75-79.

32. Garrow D, Miller S, Sinha D, et al. Endoscopic ultrasound: a metaanalysis of test performance in suspected biliary obstruction. Clin Gastroenterol Hepatol. 2007;5:616-623.

33. Tse F, Liu L, Barkun A, Armstrong D, Moayyedi P. EUS: a meta-analysis of test performance in suspected choledocholithiasis. Gastrointest Endosc. 2008;67(2):235-244.

34. Buscarini E, Buscarini L. The role of endosonography in the diagnosis of choledocholithiasis. Eur J Ultrasound. 1999;10(2-3):117-125.

35. De Waele E, Op de Beeck B, De Waele B, Delvaux G. Magnetic resonance cholangiopancreatography in the preoperative assessment of patients with biliary pancreatitis. Pancreatology. 2007;7(4):347-351.

36. Xiao B, Zhang X, Tang W, Zeng L, Zhai Z. Magnetic resonance imaging for local complications of acute pancreatitis: a pictorial review. World J Gastroenterol. 2010;16(22):2735-2742.

37. Wu B, Hwang J, Gardner T, et al. Lactated Ringer's solution reduces systemic inflammation compared with saline in patients with acute pancreatitis. Clin Gastroenterol Hepatol. 2011;9(8):710-717.

38. Aggarwal A, Manrai M, Kochhar R. Fluid resuscitation in acute pancreatitis. World J Gastroenterol. 2014;20(48):18092-18103.

39. van Dijk SM, Hallsensleben NDL, van Santvoort HC, et al. Acute pancreatitis: recent advances through randomised trials. Gut. 2017;66(11):2024-2032.

40. Haydock M, Mittal A, Wilms H, Phillips A, Petrov M, Windsor J. Fluid therapy in acute pancreatitis: anybody's guess. Ann Surg. 2013;257(2):182-188.

41. McClave S. Drivers of oxidative stress in acute pancreatitis: the role of nutrition therapy. JPEN J Parenter Enteral Nutr. 2012;36(1):24-35.

42. Al-Omran M, Albalawi Z, Tashkandi M, Al-Ansary L. Enteral versus parenteral nutrition for acute pancreatitis. Cochrane Database Syst Rev. 2010;(1):CD002837.

43. Sathiaraj E, Murthy S, Mansard M, Rao G, Mahukar S, Reddy D. Clinical trial: oral feeding with a soft diet compared with clear liquid diet as initial meal in mild acute pancreatitis. Aliment Pharmacol Ther. 2008;28(6):777-781.

44. Jacobson B, Vander Vliet M, Hughes M, Maurer R, McManus K, Banks P. A prospective, randomized trial of clear fluids versus low-fat solid diet as the initial meal in mild acute pancreatitis. Clin Gastroenterol Hepatol. 2007;5(8):946-951.

45. Moraes J, Felga G, Chebli L, et al. A full solid diet as the initial meal in mild acute pancreatitis is safe and result in shorter length of hospitalization: results from a prospective, randomized, controlled, double-blind clinical trial. J Clin Gastroenterol. 2010;44(7):517-522.

46. Chebli J, Gaburri P, De Souza A, et al. Oral refeeding in patients with mild acute pancreatitis: prevalence and risk factors of relapsing abdominal pain. J Gastroenterol Hepatol. 2005;20(9):1385-1389.

47. Wu B, Banks P. Clinical management of patients with acute pancreatitis. Gastroenterology. 2013;144(6):1272-1281.

48. Marik P, Zaloga G. Meta-analysis of parenteral nutrition versus enteral nutrition in patients with acute pancreatitis. BMJ. 2004;328(7453):1407. 
49. Windsor AC, Kanwar S, Li AG, et al. Compared with parenteral nutrition, enteral feeding attenuates the acute phase response and improve disease severity in acute pancreatitis. Gut. 1998;42(3):431-435.

50. Li Y, Yu T, Chen G, et al. Enteral nutrition within 48 hours of admission improves clinical outcomes if acute pancreatitis by reducing complications: a meta-analysis. PLoS One. 2013;8(6):e64926.

51. Bakker OJ, van Brunschot S, van Santvoort HC, et al. Early versus ondemand nasoenteric tube feeding in acute pancreatitis. $N$ Engl J Med. 2014;371:1983-1993.

52. Stimac D, Poropat G, Hauser G, et al. Early nasojejunal tube feeding versus nil-by-mouth in acute pancreatitis: a randomized clinical trial. Pancreatology. 2016;16(4):523-528.

53. Chang Y, Hua-gun F, Xiao Y, Liu J. Nasogastric or nasojejunal feeding in severe acute pancreatitis: a meta-analysis. Crit Care. 2013;17(3):R118.

54. Eatock FC, Chong P, Menezes N, et al. A randomized study of early nasogastric versus nasojejunal feeding in severe acute pancreatitis. $\mathrm{Am}$ J Gastroenterol. 2005;100(2):432-439.

55. Schmid S, Uhl W, Friess H. The role of infection in acute pancreatitis. Gut. 1999;45(2):311-316.

56. Mourad MM, Evans RP, Kalidindi V, Drorkin L, Dvorkin L, Bramhall SR. Prophylactic antibiotics in acute pancreatitis: endless debate. Ann $R$ Coll Surg Engl. 2017;99(2):107-112.

57. Villatoro E, Mulla M, Larvin M. Antibiotic therapy for prophylaxis against infection of pancreatic necrosis in acute pancreatitis. Cochrane Database Syst Rev. 2010;(5):CD002941.

58. Golub R, Siddiqi F, Pohl D. Role of antibiotics in acute pancreatitis: a meta-analysis. J Gastrointest Surg. 1998;2(6):496-503.

59. Villatoro E, Bassi C, Larvin M. Antibiotic therapy for prophylaxis against infection of pancreatic necrosis in acute pancreatitis. Cochrane Database Syst Rev. 2006;(4):CD002941.

60. Mazaki T, Ishii Y, Takayama T. Meta-analysis of prophylactic antibiotic use in acute necrotizing pancreatitis. Br J Surg. 2006;93(6):647-684.

61. Lim C, Lee W, Liew Y, Tang S, Chlebicki M, Kwa A. Role of antobiotic prophylaxis in necrotizing pancreatitis: a meta-analysis. J Gastrointest Surg. 2015;19(3):480-491.

62. Wilson C, de Moya M. Cholecystectomy for acute gallstone pancreatitis: early vs. delayed approach. Scand J Surg. 2010;99:81-85.

63. Association of Upper Gastrointestinal Surgeons. Commissioning Guide: Gallstone Disease. Royal College of Surgeons: London, UK; 2016.
64. Gurusamy K, Nagendran M, Davidson B. Early versus delayed laparoscopic cholecystectomy for acute gallstone pancreatitis. Cochrane Database Syst Rev. 2013;(9):CD010326.

65. van Baal M, Besselink M, Bakker O, et al. Timing of cholecystectomy after mild biliary pancreatitis: a systematic review. Ann Surg. 2012;255(5):860-866.

66. Dasari B, Tan C, Gurusamy K, et al. Surgical versus endoscopic treatment of bile duct stones. Cochrane Database Syst Rev. 2013;(12):CD003327

67. Al-Temimi M, Kim E, Chandrasekaran B, et al. Laparoscopic common bile duct exploration versus endoscopic retrograde cholangiapancreatography for choledocholithiasis found at time of laparoscopic cholecystectomy: analysis of a large integrated health care system database. Am J Surg. 2017;214(6):1075-1079.

68. Griniatsos J, Karvounis E, Isla A. Early versus delayed singlestage laparoscopic eradication for both gallstones and common bile duct stones in mild acute biliary pancreatitis. Am Surg. 2005;71(8):682-686.

69. Isla A, Griniatsos J, Rodway A. Single-stage definitive laparoscopic management in mild acute biliary pancreatitis. $J$ Laparoendosc Adv Surg Tech A. 2003;13(2):77-81.

70. Meyer C, Le J, Rohr S, Duclos B, Reimund J, Baumann R. Management of common bile duct stones in a single operation combining laparoscopic cholecystectomy and peroperative endoscopic sphincterotomy. J Hepatobiliary Pancreat Surg. 2002;9(2):196-200.

71. Tse F, Yuan Y. Early routine endoscopic retrograde cholangiopancreatography strategy versus early conservative management strategy in acute gallstone pancreatitis. Cochrane Database Syst Rev. 2012;(5):CD009779.

72. Karakayali F. Surgical and interventional management of complications caused by acute pancreatitis. World J Gastroenterol. 2014;20(37):13412-13423.

73. Wronski M, Cebulski W, Witkowski B, et al. Comparison between minimally invasive and open surgical treatment in necrotizing pancreatitis J Surg Res. 2017;210:22-31.

74. Werner J, Feuerbach S, Uhl W, Buchler M. Management of acute pancreatitis: from surgery to interventional intensive care. Gut 2005;54(3):426-436.
Journal of Inflammation Research

\section{Publish your work in this journal}

The Journal of Inflammation Research is an international, peer-reviewed open access journal that welcomes laboratory and clinical findings on the molecular basis, cell biology and pharmacology of inflammation including original research, reviews, symposium reports, hypothesis formation and commentaries on: acute/chronic inflammation; mediators of

\section{Dovepress}

inflammation; cellular processes; molecular mechanisms; pharmacology and novel anti-inflammatory drugs; clinical conditions involving inflammation. The manuscript management system is completely online and includes a very quick and fair peer-review system. Visit http://www.dove press.com/testimonials.php to read real quotes from published authors. 